\title{
Organization of the International Academy of Sportology
}

\author{
Executive Council \\ MORI Yoshiro (President, The Tokyo Organizing Committee of the Olympic and Paralympic \\ Games) \\ TAKAKU Fumimaro (President, The Japanese Association of Medical Sciences) \\ OGAWA Hideoki (CEO, Juntendo University) \\ KAWAMORI Ryuzo (Director, Sportology Center, Juntendo University) \\ Honorary President \\ OGAWA Hideoki (CEO, Juntendo University) \\ Advisers \\ Pierre LEFEVBRE (Emeritus Professor, University of Liège, Former President of International \\ Diabetes Federation) \\ Mladen VRANIC (Emeritus Professor, University of Toronto) \\ KOMINAMI Eiki (President, Juntendo University) \\ ARAI Hajime (Dean, Juntendo University Faculty of Medicine) \\ KANOU Minoru (Dean, Juntendo University Faculty of Health and Sports Science)
}

\section{Local Organizing Committee of the $2^{\text {nd }}$ Congress International Academy of Sportology}

\section{Chairman}

KAWAMORI Ryuzo (Director, Sportology Center, Juntendo University)

\section{Vice-chairman}

DAIDA Hiroyuki (Professor, Juntendo University)

KANEKO Kazuo (Professor, Juntendo University)

\section{Secretary General}

WATADA Hirotaka (Professor, Juntendo University)

\section{Secretary}

TAMURA Yoshifumi (Associate Professor, Juntendo University) 